\title{
Producto interno bruto provincial (PIB) y el mercado laboral en Panamá durante
} el periodo 2007-2016.

Provincial gross domestic product (GDP) and the labor market in Panama during the period 2007-2016.

\section{Jimmy Perez1， Jaime M. González2}

1. Licenciatura en Mercadeo y Negocios Internacionales, Centro Regional de Coclé, Universidad Tecnológica de Panamá. agustinperez27@hotmail.com, https://orcid.org/0000-0002-0924-3354

2. Profesor Regular (Universidad de Panamá); Lic. En Economía, Máster en Economía y Desarrollo. Jaime_manuel@ hotmail.com; https://orcid.org/0000-0002-7718-1896

Págs.: 70 - 85

Recibido: 4/8/2020 Aprobado: 18/8/2020

\section{Resumen}

En el análisis de las variables económicas y su relación con el mercado laboral se utiliza en este artículo la teoría de crecimiento de Solow. Aplicando descripciones matemáticas y gráficas teóricas del comportamiento de las variables de producción y el capital humano y físico de un país. Esta teoría proporcionó los elementos fundamentales para la búsqueda de datos provinciales y comarcales en Panamá. El Producto Interno Bruto (PIB) y la Población Ocupada (PO) y el Empleo Informal (EI). La relación de estas variables se produjo por medio de estructuras espaciales y con una técnica econométrica llamada Datos de paneles. Con esta técnica simulamos tres (3) modelos, Regresión Agrupada (pooled ols), Efectos Aleatorios (random effects, Efectos Fijos (fixed effects) y que nos proporciona una visión de medición y de aceptación con el modelo de Efectos Fijos (fixed effects) en Panamá.

Palabra Claves: Modelo de SOLOW, Datos de Panel, Crecimiento Económico 


\begin{abstract}
In the analysis of economic variables and their relationship with the labor market, Solow's growth theory is used in this article. Applying mathematical descriptions and theoretical graphs of the behavior of production variables and the human and physical capital of a country.

This theory provided the fundamental elements for searching provincial and county data in Panama. The Gross Domestic Product (GDP) and the Employed Population (PO) and Informal Employment (EI). The relationship of these variables was produced by means of spatial structures and with an econometric technique called Panel Data. With this technique we simulate three (3) models, Pooled ols, Random Effects, Fixed Effects and that gives us a measurement and acceptance view with the Fixed Effects model in Panama.
\end{abstract}

\title{
Key Words: SOLOW Model, Panel Data, Economic Growth
}

\section{Introducción}

Es necesario analizar las causas y efectos del crecimiento panameño que tiene mucha homogeneidad con economías mundiales y regionales estableciendo un modelo económico que debe ser revisado a partir de nuevas metodologías y técnicas de manejo de información global. Hay una variación enorme en el ingreso per cápita entre las economías. Los países más pobres tienen ingresos per cápita que son inferiores al $5 \%$ de los ingresos per cápita de los países más ricos. Las tasas de crecimiento económico varían en forma importante entre países. Las tasas de crecimiento no son necesariamente constantes en el transcurso del tiempo. La posición relativa de un país en la distribución mundial del ingreso per cápita no es inmutable. Los países pueden cambiar de ser "pobres" a ser "ricos" y viceversa. El crecimiento en la producción y el crecimiento en el volumen del comercio internacional están estrechamente relacionados. Tanto los trabajadores calificados como los no calificados tienden a emigrar de países o regiones pobres a los ricos.

Por tal razón, utilizaremos el modelo de crecimiento de Solow para orientar nuestras respuestas al estudio de la economía panameña. 


\section{Materiales y Métodos}

\section{Modelo de Solow}

De acuerdo a Charles I. Jones (2000), el modelo de Solow está construido alrededor de dos ecuaciones: función de producción, Ecuación de capital.

A fin de simplificar el modelo se agrupan estos insumos en dos categorías, capital K y trabajo L, y el producto se representa como Y. se supone que la función de producción tiene la forma de Cobb-Douglas y se determina por

$$
Y=F(K, L)=K^{\alpha} L^{1-\alpha}
$$

$\alpha$, es algún número entre 0 y 1 . Obsérvese que esta función de producción muestra rendimientos constantes a escala si se duplican todo insumos, la producción se duplicará exactamente las empresas en esta economía pagan a los trabajadores un salario, W, por cada unidad de trabajo y pagan $\mathrm{r}$ con el fin de arrendar una unidad de capital por un periodo.

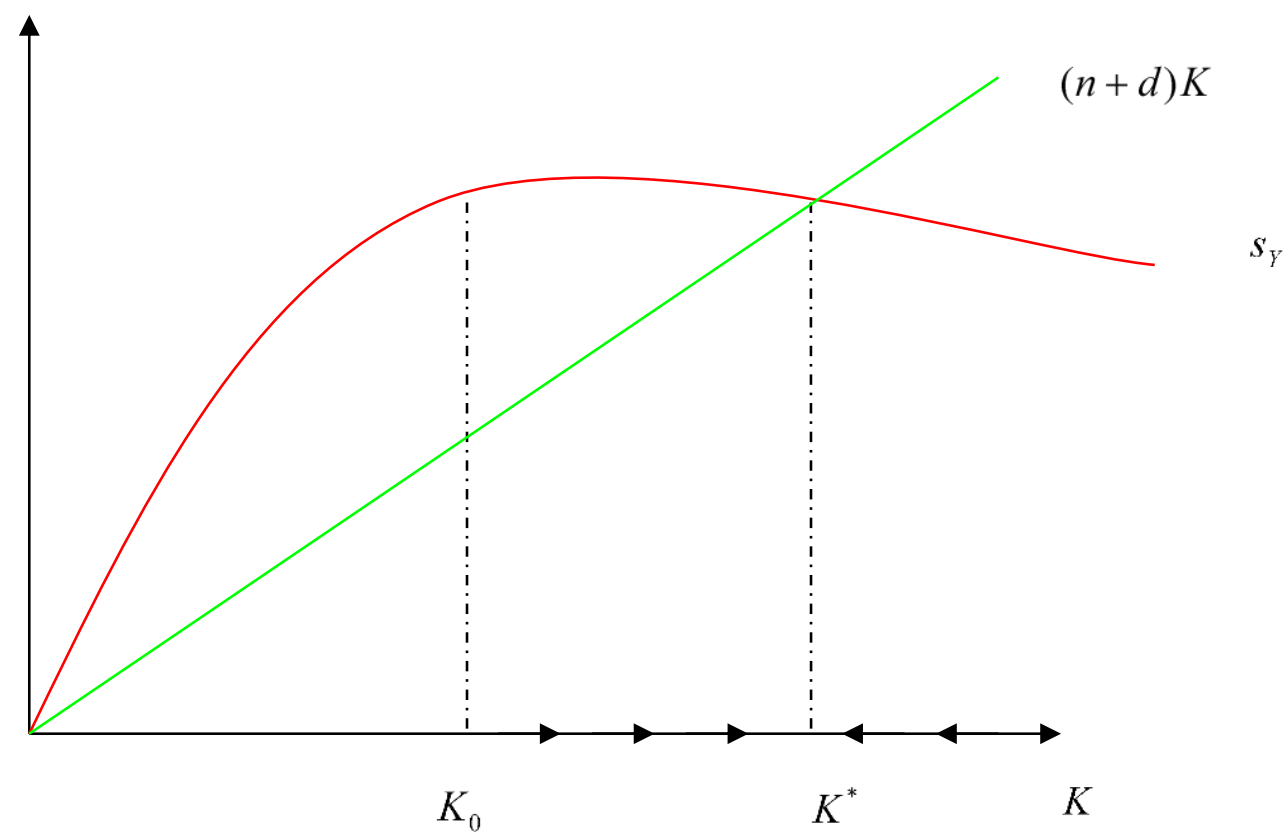

\section{Figura $N^{\circ}$ 1: El Diagrama Básico de Solow}


Al normalizar el precio de la producción en nuestra economía a la unidad, las empresas maximizadoras de beneficios solucionan el siguiente problema.

$$
\max _{K, L} F(K, L)-r K-w L
$$

De acuerdo con las condiciones de primer orden para este problema, las empresas contratarán trabajo hasta que el producto marginal del trabajo sea igual a los salarios, y arrendarán capital hasta que el producto marginal del capital sea igual al precio de arrendamiento (Ver fig. 1)

$$
\begin{gathered}
W=\frac{\partial F}{\partial L}=(1-\alpha) \frac{Y}{L} \\
r=\frac{\partial F}{\partial K}=\alpha \frac{Y}{K}
\end{gathered}
$$

Obsérvese que $W L+r K=Y$. Es decir, los pagos a los insumos (pagos a los factores) agotan por completo el valor de la producción fabricada, por lo que no hay ganancias económicas que obtener.

La producción por trabajador a la producción per-cápita: se puede adquirir a partir de la función de producción determinando que la producción por trabajador, $y \equiv \frac{Y}{L}$ y el capital por trabajador, $k \equiv \frac{K}{L}$

$$
y=k^{\alpha}
$$

La segunda ecuación clave del modelo de Solow es la que describe cómo se acumula el capital. La ecuación de la acumulación del capital se expresa mediante 
GUACAMAYA

$$
\dot{K}=s Y-d K
$$

El cambio en la existencia de capital $\dot{K}$, es igual a la cantidad de inversión bruta $s Y$, menos la cantidad de depreciación que ocurre durante el proceso de producción $d K$. Ahora se examinarán estos tres términos en forma más detallada. (Ver fig. 2)

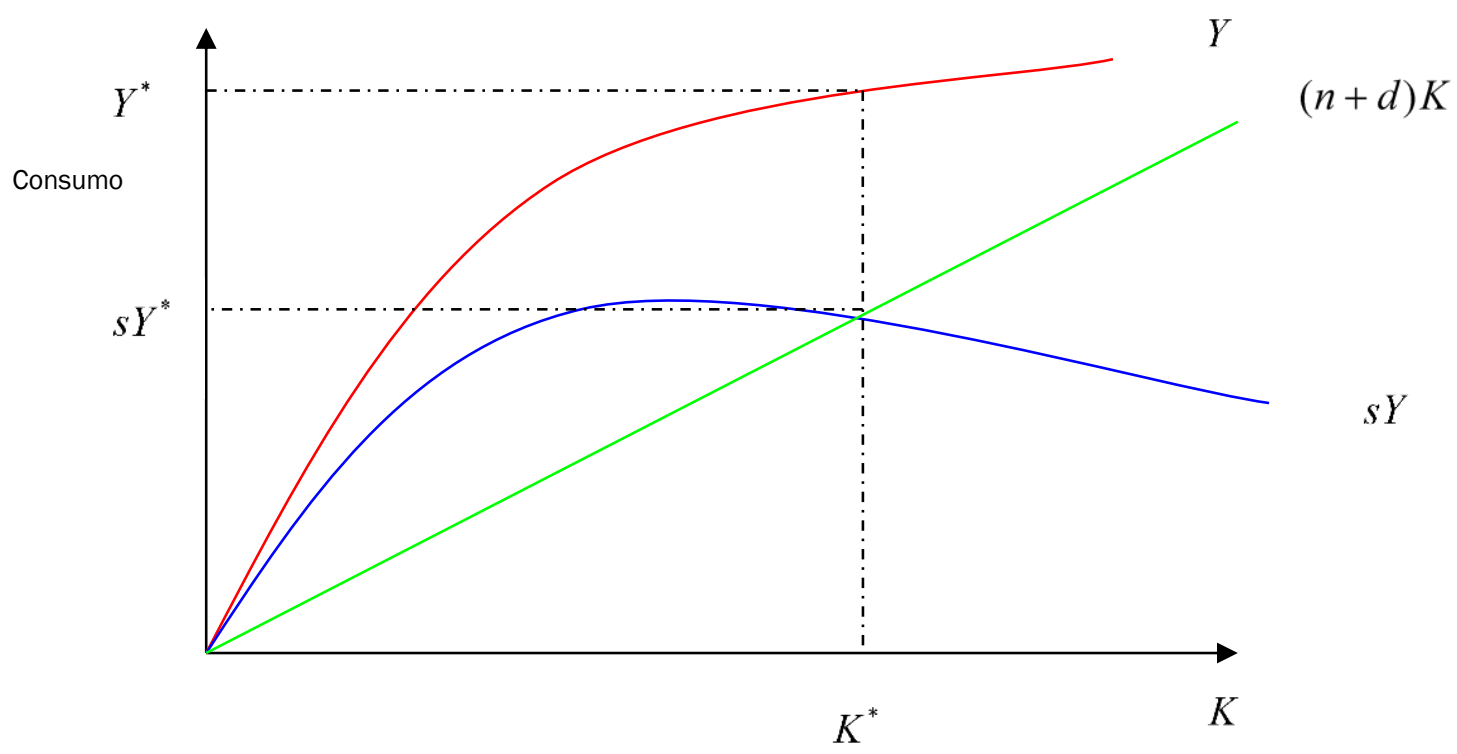

Figura $N^{\circ}$ 2: El Diagrama de Solow y la Función de Producción

El término al lado izquierdo de la ecuación ( 6 ) es la versión continua de tiempo de $K_{t-1}-K_{t}$, es decir, el cambio en las existencias de capital por periodo usamos la notación del punto para indicar una derivada con relación al tiempo:

$$
\dot{K} \equiv \frac{d K}{d t}
$$

El segundo término de la ecuación (sY) representa la inversión bruta. De acuerdo con Solow, se supone que los trabajadores/consumidores ahorran una parte constante, S, de su ingreso combinado de salarios y arrendamiento, $Y=w L+r K$ 


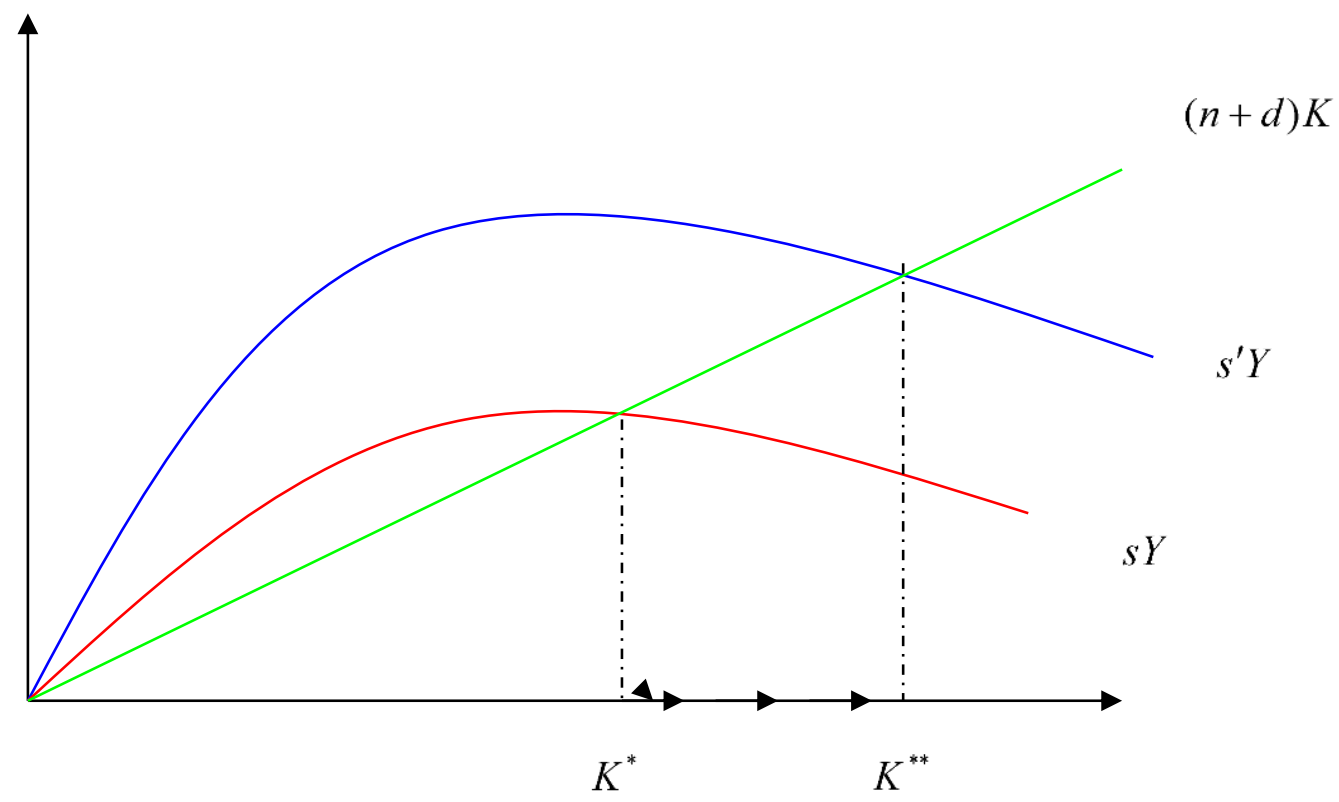

Figura $\mathrm{N}^{\circ}$ 3: Un Aumenta en la Tasa de Inversión

El tercer término de la ecuación $(\mathrm{dK})$ refleja la depreciación de la existencia de capital que ocurre durante la producción. La forma funcional estándar utilizada aquí implica que en cada periodo se deprecia una parte constante, d, de la existencia de capital (con independencia de cuanta producción se realice)

A continuación, se presentan dos ejemplos de este truco matemático. (Ver fig. 3)

$$
\begin{gathered}
k \equiv \frac{K}{L} \Rightarrow \log k=\log K-\log L \\
\frac{\dot{k}}{K}=\frac{\dot{K}}{K}-\frac{\dot{L}}{L} \\
Y \equiv K^{\alpha} \Rightarrow \log Y=\alpha \log K \\
\Rightarrow \frac{\dot{Y}}{Y}=\alpha \frac{\dot{K}}{K}
\end{gathered}
$$


El crecimiento exponencial se puede observar de la relación.

$$
L(t)=L_{0} e^{n t}
$$

Si se toman los logaritmos de Y se diferencia esta ecuación, ¿Qué se tiene?

$$
\begin{aligned}
\frac{\dot{K}}{K} & =s \frac{Y}{K}-n-d \\
& =\frac{s Y}{k}-n-d
\end{aligned}
$$

Esto da como resultado la ecuación de la acumulación de capital en término sor trabajador:

$$
\dot{K}=s y-(n+d) k
$$

La inversión por trabajador ${ }^{s y}$, aumenta k, mientras que la depreciación por trabajador, $d k$, disminuye k. El término que es nuevo en esta ecuación es una reducción de k, debido al crecimiento de la población, el término $n k$. En cada periodo, hay $n L_{\text {nuevos }}$ trabajadores que no se encontraban ahí durante el último periodo. Si no hubo nueva inversión ni depreciación, el capital por trabajador disminuiría es exactamente $n k$, como se puede observar estableciendo a $\dot{K}$, como cero. (Ver fig. 4 ) 


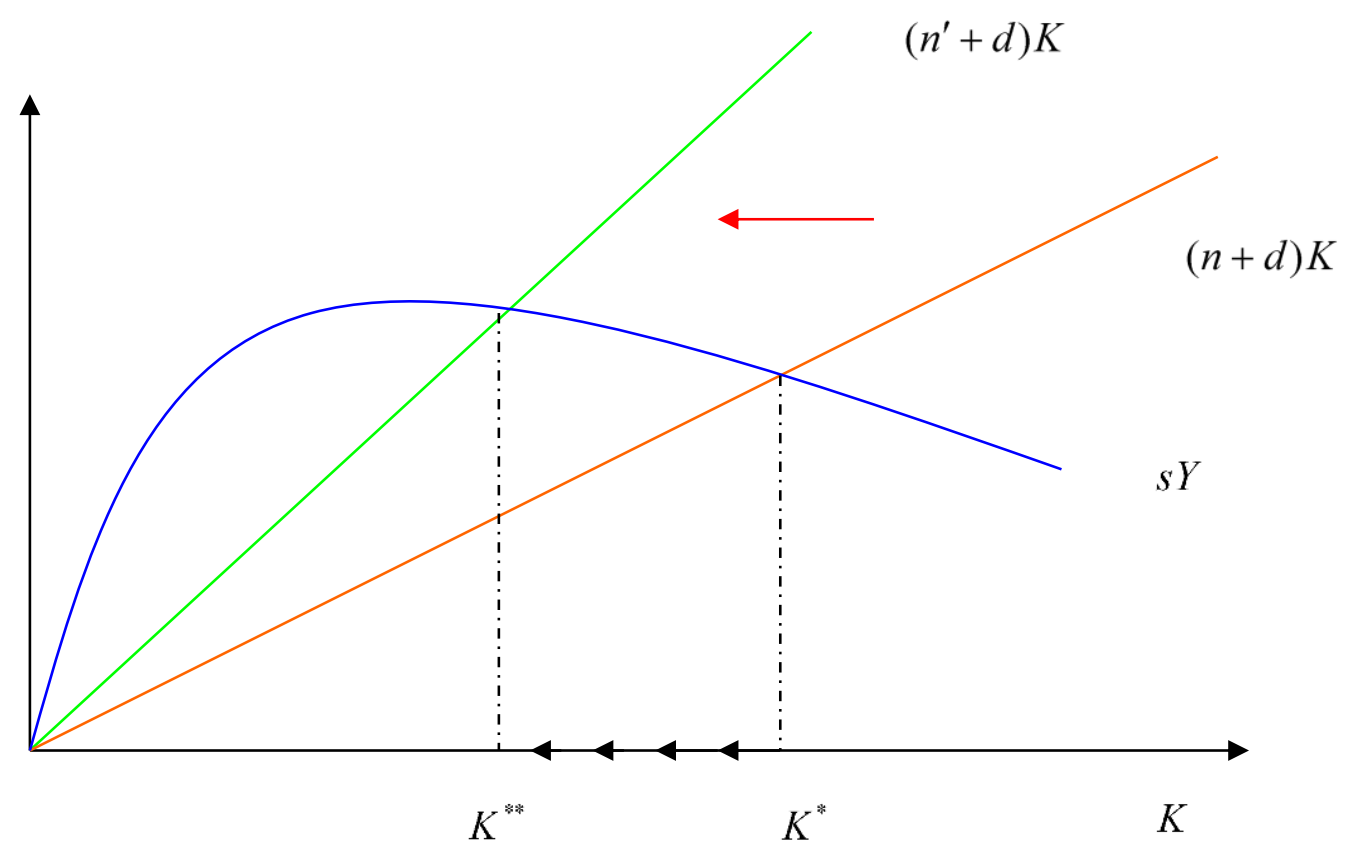

Figura $N^{\circ}$ 4: Un Aumento en el Crecimiento de la Población

\section{Resultados y Discusión}

De acuerdo a Herrera, A., \& González, J. (2018) y Him, R., Ortega, L., \& González, J. (2019). El propósito de esta sesión es introducir brevemente algunos comandos de Stata 12.0 para especificar modelos econométricos con datos tipo panel. La sesión es práctica, por lo que utilizaremos la base Base de Datos (PIB-PO-EI) 2.dta para estimar el impacto de las variables del Producto Interno Bruto Provincial (PIB) y el mercado Laboral (Población Ocupada (PO) y Empleo Informal (EI)) en Panamá durante el periodo 20072016.

Pérez, J., Ashaw, M., Henríquez, T., \& González, J. (2019), durante la sesión recurriremos a comandos que no están cargados en Stata 12.0. Es importante entonces que antes de iniciar escribas en la línea de comando (mientras estás conectado a Internet) las siguientes indicaciones (Ver Tabla $\mathrm{N}^{\circ} 1$ ) 
TABLA $\mathbf{N}^{\circ}$ 1:

BASE DE DATOS PRODUCCIÓN INTERNA BRUTA (PIB), POBLACIÓN OCUPADA Y EMPLEO INFORMAL, PROVINCIAL, AÑOS: 2007-2016.

\begin{tabular}{|c|c|c|c|c|c|}
\hline PROV & $\mathrm{i}$ & $\mathrm{t}$ & $\mathrm{PIB}$ & $\mathrm{PO}$ & EI \\
\hline Bocas del Toro & 1 & 2007 & 263 & 16,066 & 8,998 \\
\hline Coclé & 2 & 2007 & 674 & 61,046 & 37,023 \\
\hline Colón & 3 & 2007 & 2,860 & 85,086 & 33,872 \\
\hline Chiriquí & 4 & 2007 & 1,362 & 112,179 & 60,530 \\
\hline Darién & 5 & 2007 & 83 & 6,936 & 4,348 \\
\hline Herrera & 6 & 2007 & 312 & 31,066 & 17,679 \\
\hline Los Santos & 7 & 2007 & 288 & 25,768 & 14,633 \\
\hline Panamá & 8 & 2007 & 14,926 & 667,992 & 254,211 \\
\hline Veraguas & 9 & 2007 & 528 & 49,683 & 26,651 \\
\hline Bocas del Toro & 1 & 2008 & 377 & 18,120 & 9,576 \\
\hline Coclé & 2 & 2008 & 704 & 62,413 & 35,562 \\
\hline Colón & 3 & 2008 & 3,167 & 93,859 & 36,036 \\
\hline Chiriquí & 4 & 2008 & 1,480 & 116,411 & 65,396 \\
\hline Darién & 5 & 2008 & 94 & 6,328 & 3,090 \\
\hline Herrera & 6 & 2008 & 335 & 31,243 & 16,836 \\
\hline Los Santos & 7 & 2008 & 324 & 28,585 & 16,226 \\
\hline Panamá & 8 & 2008 & 16,357 & 716,285 & 266,220 \\
\hline Veraguas & 9 & 2008 & 557 & 49,268 & 24,804 \\
\hline Bocas del Toro & 1 & 2009 & 366 & 18,068 & 8,938 \\
\hline Coclé & 2 & 2009 & 658 & 62,123 & 36,632 \\
\hline Colón & 3 & 2009 & 3,097 & 88,531 & 32,086 \\
\hline Chiriquí & 4 & 2009 & 1,624 & 115,575 & 63,372 \\
\hline Darién & 5 & 2009 & 86 & 6,910 & 4,716 \\
\hline Herrera & 6 & 2009 & 385 & 34,788 & 18,515 \\
\hline Los Santos & 7 & 2009 & 272 & 29,242 & 16,175 \\
\hline Panamá & 8 & 2009 & 16,634 & 722,014 & 260,872 \\
\hline Veraguas & 9 & 2009 & 565 & 48,828 & 24,501 \\
\hline Bocas del Toro & 1 & 2010 & 340 & 17,908 & 8,084 \\
\hline Coclé & 2 & 2010 & 693 & 63,502 & 35,701 \\
\hline Colón & 3 & 2010 & 3,270 & 91,598 & 34,932 \\
\hline Chiriquí & 4 & 2010 & 1,655 & 117,994 & 61,070 \\
\hline Darién & 5 & 2010 & 89 & 6,142 & 3,918 \\
\hline Herrera & 6 & 2010 & 385 & 36,304 & 18,904 \\
\hline Los Santos & 7 & 2010 & 283 & 28,288 & 15,077 \\
\hline Panamá & 8 & 2010 & 17,772 & 740,049 & 264,177 \\
\hline Veraguas & 9 & 2010 & 577 & 49,962 & 23,940 \\
\hline Bocas del Toro & 1 & 2011 & 354 & 24,589 & 10,817 \\
\hline Coclé & 2 & 2011 & 734 & 65,673 & 33,416 \\
\hline Colón & 3 & 2011 & 3,620 & 92,270 & 34,271 \\
\hline Chiriquí & 4 & 2011 & 1,595 & 122,579 & 59,547 \\
\hline Darién & 5 & 2011 & 94 & 8,614 & 5,001 \\
\hline Herrera & 6 & 2011 & 392 & 37,895 & 17,604 \\
\hline Los Santos & 7 & 2011 & 299 & 31,821 & 16,184 \\
\hline Panamá & 8 & 2011 & 20,190 & 800,416 & 250,182 \\
\hline Veraguas & 9 & 2011 & 618 & 48,916 & 18,805 \\
\hline Bocas del Toro & 1 & 2012 & 369 & 28,731 & 14,181 \\
\hline Coclé & 2 & 2012 & 783 & 76,465 & 37,540 \\
\hline Colón & 3 & 2012 & 3,810 & 96,759 & 34,646 \\
\hline Chiriquí & 4 & 2012 & 1,758 & 127,554 & 62,000 \\
\hline Darién & 5 & 2012 & 94 & 8,795 & 4,840 \\
\hline Herrera & 6 & 2012 & 415 & 39,846 & 19,108 \\
\hline Los Santos & 7 & 2012 & 315 & 33,732 & 17,597 \\
\hline Panamá & 8 & 2012 & 22,425 & 832,122 & 257,990 \\
\hline Veraguas & 9 & 2012 & 646 & 52,969 & 22,222 \\
\hline Bocas del Toro & 1 & 2013 & 415 & 32,527 & 17,356 \\
\hline Coclé & 2 & 2013 & 871 & 70,583 & 34,535 \\
\hline Colón & 3 & 2013 & 3,575 & 95,063 & 31,244 \\
\hline Chiriquí & 4 & 2013 & 1,806 & 135,943 & 70,341 \\
\hline Darién & 5 & 2013 & 99 & 10,084 & 6,150 \\
\hline Herrera & 6 & 2013 & 442 & 37,568 & 17,789 \\
\hline Los Santos & 7 & 2013 & 333 & 33,679 & 17,072 \\
\hline Panamá & 8 & 2013 & 24,412 & 874,602 & 81,300 \\
\hline Veraguas & 9 & 2013 & 778 & 58,434 & 26,756 \\
\hline Bocas del Toro & 1 & 2014 & 436 & 33,751 & 17,746 \\
\hline Coclé & 2 & 2014 & 936 & 71,174 & 36,554 \\
\hline Colón & 3 & 2014 & 3,498 & 96,747 & 34,859 \\
\hline Chiriquí & 4 & 2014 & 1,864 & 134,474 & 67,899 \\
\hline Darién & 5 & 2014 & 97 & 11,928 & 7,365 \\
\hline
\end{tabular}

http://revistas.up.ac.pa/index.php/guacamaya 
GUACAMAYA

\begin{tabular}{|c|c|c|c|c|c|}
\hline Herrera & 6 & 2014 & 511 & 39,806 & 18,906 \\
\hline Los Santos & 7 & 2014 & 360 & 36,043 & 18,145 \\
\hline Panamá & 8 & 2014 & 25,968 & 891,010 & 299,664 \\
\hline Veraguas & 9 & 2014 & 727 & 59,622 & 27,925 \\
\hline Bocas del Toro & 1 & 2015 & 471 & 35,231 & 19,320 \\
\hline Coclé & 2 & 2015 & 925 & 82,409 & 44,264 \\
\hline Colón & 3 & 2015 & 4,361 & 96,409 & 35,825 \\
\hline Chiriquí & 4 & 2015 & 2,039 & 131,385 & 60,116 \\
\hline Darién & 5 & 2015 & 95 & 11,512 & 7,550 \\
\hline Herrera & 6 & 2015 & 477 & 41,198 & 19,337 \\
\hline Los Santos & 7 & 2015 & 368 & 39,389 & 21,430 \\
\hline Panamá & 8 & 2015 & 26,823 & 915,605 & 319,785 \\
\hline Veraguas & 9 & 2015 & 779 & 63,845 & 28,346 \\
\hline Bocas del Toro & 1 & 2016 & 485 & 28,920 & 15,873 \\
\hline Coclé & 2 & 2016 & 948 & 81,866 & 44,275 \\
\hline Colón & 3 & 2016 & 4,413 & 97,064 & 35,406 \\
\hline Chiriquí & 4 & 2016 & 2,101 & 130,885 & 62,073 \\
\hline Darién & 5 & 2016 & 96 & 9,996 & 6,685 \\
\hline Herrera & 6 & 2016 & 484 & 40,388 & 18,418 \\
\hline Los Santos & 7 & 2016 & 372 & 38,682 & 20,917 \\
\hline Panamá & 8 & 2016 & 28,493 & 683,508 & 233,331 \\
\hline Veraguas & 9 & 2016 & 794 & 65,885 & 28,897 \\
\hline
\end{tabular}

Nota. Fuente: Estadísticas laborales, Cuentas Nacionales, Instituto Nacional de estadísticas y Censos Contraloría General de la República de Panamá.

Establecido en la metodología de González, J. (2017). Tomamos la base de datos provinciales se puede extraer que se analizan 90 datos, que la media del Producto Interno Bruto (PIB), está en 3262 millones de dólares, con una desviación estándar es de 6714 millones. La población ocupada (PO) se describe con una media de 136701.3 y una desviación estándar de 234,608.7. De igual forma, el empleo Informal (EI) describe una media 51,474 y una desviación estándar de 74828.9. Debemos tomar en consideración que la nube de puntos es positiva. (Ver Tabla $\mathbf{N}^{\circ} \mathbf{2}$ )

Tabla $\mathbf{N}^{\circ}$ 2:

Datos descriptivos

\begin{tabular}{llll}
\hline $\begin{array}{l}\text { Variable } \\
\text { Min }\end{array}$ & $\begin{array}{l}\text { Obs } \\
\text { Max }\end{array}$ & Mean & Std. Dev. \\
\hline pib | & 90 & 3262.278 & 6714.399 \\
83 & 28493 & & \\
po | & 90 & 136701.3 & 234608.7 \\
6142 & 915605 & & \\
ei $\mid$ & $90 \quad 51474.51$ & 74828.9 \\
3090 & \multicolumn{2}{l}{319785} & \\
\hline
\end{tabular}

Nota: se utilizó en stata 12, summarize pib po ei. Fuente: Javier Aparicio y Javier Márquez (2005). DiAgNóSTICO Y ESPECIFICACIÓN DE MODELOS PANEL EN STATA 8.0, División de Estudios Políticos, CIDE, México. 


\section{Regresión agrupada (pooled ols)}

Javier Aparicio y Javier Márquez (2005), El enfoque más simple de analizar datos tipo panel es omitir las dimensiones del espacio y el tiempo de los datos agrupados y sólo calcular la regresión MCO usual. Este modelo se expresa como:

$$
Y_{i t}=\alpha+\beta_{1} X_{1 i t}+e_{i t}
$$

Donde i significa la i-ésima unidad transversal (estado) y t el tiempo t (año). Si tratamos de explicar la variable spend con las variables independientes de la clase pasada, basta con que indiquemos en la ventana de comandos de Stata (Ver Tabla $\mathbf{N}^{\circ}$ 2)

\section{Tabla $\mathbf{N}^{\circ} 3$}

\section{Resultado de Regresión agrupada}

\begin{tabular}{|c|c|c|c|c|}
\hline $\begin{array}{l}\text { pib | } \\
\text { [95\% }\end{array}$ & Std. Err. & $\mathrm{t}$ & & $>|t|$ \\
\hline .0316293 & .0020888 & 15.14 & & 000 \\
\hline .0357809 & & & & \\
\hline $\begin{array}{lc}\mathrm{ei} \mid & -.0121795 \\
.0251961 & .0008371\end{array}$ & .0065489 & -1.86 & 0.066 & _ \\
\hline $\begin{array}{lc}\text { cons | } & -434.5542 \\
808.7911 & -60.31735\end{array}$ & 188.285 & -2.31 & 0.023 & - \\
\hline
\end{tabular}

Nota: se utilizó en stata 12, reg pib po ei. Fuente: Javier Aparicio y Javier Márquez (2005). DiAgNóstico Y ESPECIFICACIÓN DE MODELOS PANEL EN STATa 8.0, División de Estudios Políticos, CIDE, Mexico. Number of obs $=90 ; \mathrm{F}(2,87)=918.85$; Prob $>\mathrm{F}=0.0000 ; \mathrm{R}$-squared $=0.9548 ;$ Adj R-squared $=$ 0.9538; $\mathrm{F}(2, \quad 87)=918.85 ;$ Prob $>\mathrm{F}=0.0000$

\section{Efectos aleatorios (random effects)}

La ecuación (13) supone que el intercepto de la regresión es la misma para todas las unidades transversales. Sin embargo, es muy probable que necesitemos controlar el carácter "individual" de cada estado. El modelo de efectos aleatorios permite suponer que cada unidad transversal tiene un intercepto diferente. Este modelo se expresa como:

$$
Y_{i t}=\alpha_{i}+\beta_{1} X_{1 i t}+e_{i t}
$$


Donde $\alpha_{i}=\alpha+u_{i}$. Es decir, en vez de considerar a $\alpha$ como fija, suponemos que es una variable aleatoria con un valor medio $\alpha$ y una desviación aleatoria $u_{i}$ de este valor medio. Sustituyendo $\alpha_{i}=\alpha+u_{i}$ en (2) obtenemos:

$$
Y_{i t}=\alpha+\beta_{1} X_{1 i t}+u_{i}+e_{i t}
$$

Stata estima el modelo de efectos aleatorios con el comando xtreg, re. En nuestro ejemplo, indicamos en la ventana de comandos (Ver Tabla $\left.\mathbf{N}^{\circ} \mathbf{3}\right)$

\section{Tabla $\mathbf{N}^{\circ} 4$}

\section{Resultado de regresion efectos aleatorios}

\begin{tabular}{lllll}
\hline pib $\mid \begin{array}{c}\text { Coef. } \\
\text { Conf. Interval] }\end{array}$ & Std. Err. & $\mathrm{z}$ & $\mathrm{P}>|\mathrm{z}|$ & {$[95 \%$} \\
\hline po $\mid .0309598$ & .0020732 & 14.93 & 0.000 & \\
.0268963 .0350232 & & & & \\
ei $\mid-.0099591$ & .0063823 & -1.56 & 0.119 & -.0224681 \\
.00255 & & & & \\
$\begin{array}{l}\text { cons } \mid-457.3248 \\
-14.19028\end{array}$ & 226.0932 & -2.02 & 0.043 & -900.4593 \\
\end{tabular}

Nota: se utilizó en stata 12, xtreg pib po ei, re; Fuente: Javier Aparicio y Javier Márquez (2005). DiAGNÓSTICO Y ESPECIFICACIÓN DE MODELOS PANEL EN STATA 8.0, División de Estudios Políticos, CIDE, Mexico. NUMBER OF OBS $=90 ;$ NUMBER OF GROUPS $=9$; R-SQ: WITHIN $=0.3437$; BETWEEN $=0.9899 ;$ OVERALL $=0.9547$; RHO 05964801 (FRACTION OF VARIANCE DUE TO UI); WALD CHI2 $(2)=1237.77$; CORR(U_I, X) $=0$ (ASSUMED) PROB $>\mathrm{CHI} 2=0.0000$

\section{Efectos fijos (fixed effects)}

Otra manera de modelar el carácter "individual" de cada estado es a través del modelo de efectos fijos. Este modelo no supone que las diferencias entre estados sean aleatorias, sino constantes o "fijas"-y por ello debemos estimar cada intercepto $u_{i}$ ¿ ¿Cómo podemos permitir que el intercepto varíe con respecto a cada estado? Una manera es la 
técnica de "las variables dicotómicas de intersección diferencial", que se expresa de la siguiente manera (1):

$$
Y_{i t}=v_{i}+\beta_{1} X_{1 i t}+e_{i t}
$$

Donde ${ }^{v_{i}}$ es un vector de variables dicotómicas para cada estado. El modelo de efectos fijos puede ejecutarse en Stata con el comando:

El cual estima una dummy para cada estado. Una opción más sencilla es el comando xtreg (Ver Tabla $\left.\mathbf{N}^{\circ} \mathbf{5}\right)$

Tabla $\mathbf{N}^{\circ} 5$

Resultado de regresión efectos fijos

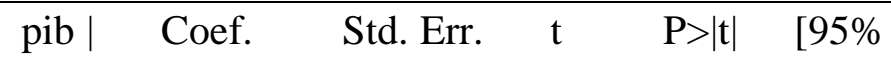

Conf. Interval]

$\begin{array}{llllll}\text { po | } & .0313335 & 0048698 & 6.43 & 0.000 \\ .0216405 \quad .0410265 & & & & \\ \text { ei | -.0051872 .0069562 } & -0.75 & 0.458 & - \\ .0190333 .0086588 & & & & \\ \text { cons | }-754.041 \quad 773.0123 & -0.98 & 0.332 & - \\ 2292.683 \quad 784.6015 & & & & \end{array}$

Nota: Se utilizó en stata, xtreg pib po ei, fe ; Fuente: Javier Aparicio y Javier Márquez (2005). DiAgNóstico Y ESPECIFICACIÓN DE MODELOS PANel en Stata 8.0, División de Estudios Políticos, CIDE, Mexico. NUMBER OF OBS = 90; NUMBER OF GROUPS $=9$; R-SQ: WITHIN $=0.3476$; BETWEEN $=$ $0.9892 ;$ OVERALL $=0.9542 ;$ RHO $\quad .28448748$ (FRACTION OF VARIANCE DUE TO UI); $\mathrm{F}(2,79)=21.04$; $\mathrm{CORR}\left(\mathrm{U} \_\mathrm{I}, \mathrm{XB}\right)=-0.5288 \quad \mathrm{PROB}>\mathrm{F}=$ 0.0000

¿Cuál de los modelos (13) y (16) es mejor? En relación con el modelo (16), el (13) es un modelo restringido, pues asume un intercepto común para todos los estados (es decir, no incluye variables dicotómicas estatales). Por lo tanto, podemos utilizar una prueba $\mathrm{F}$ restrictiva para contestar la cuestión. La hipótesis nula es que $v_{1}=v_{2}=\ldots=v_{i}=0$ sea, que todas las variables dicotómicas estatales son iguales cero). Si la prueba se

\footnotetext{
${ }^{1}$ Como repasamos en clase, utilizar variables dicotómicas conduce al mismo resultado que si restamos a cada observación la media de cada estado (demeaning the data).
} 
rechaza, significa que al menos algunas variables dicotómicas sí pertenecen al modelo, y por lo tanto es necesario utilizar el método de efectos fijos. La prueba $\mathrm{F}$ de significancia de los efectos fijos se reporta automáticamente con el comando xtreg, fe.

\section{Estimación resumen de modelos de datos de Paneles}

En la tabla $\mathrm{N}^{\circ}$ 6, se observa los parámetros econométricos y de medición para la decisión del modelo de predicción entre el producto Interno Bruto (PIB) y la Población Ocupada (PO) en Panamá.

Tabla $\mathbf{n}^{\circ}$ 6:

Estimación con datos panel

\begin{tabular}{lccc}
\hline & $\begin{array}{c}\text { Modelo 1: } \\
\text { pooled }\end{array}$ & $\begin{array}{c}\text { Modelo 2: } \\
\text { random efects }\end{array}$ & $\begin{array}{c}\text { Modelo 3: } \\
\text { fixed effects }\end{array}$ \\
\hline PO & $0.3162^{* *}$ & $0.3095^{* *}$ & $126.494^{* *}$ \\
EI & {$[0.0020]$} & {$[0.00207]$} & {$[54.539]$} \\
& -0.012179 & -0.00995 & -3.506 \\
Constant & {$[0.0065]$} & {$[0.006382]$} & {$[17.439]$} \\
& -433.55 & -457.3248 & -491.471 \\
Observations & {$[188.285]$} & {$[226.093]$} & {$[85.470]$} \\
R-squared & 90 & 90 & 90 \\
Number of stcode & 0.95 & 0.98 & 0.9892 \\
Standard errors in brackets & 10 & 10 \\
\hline
\end{tabular}

Nota: Se recogen los tres modelos; Fuente: Javier Aparicio y Javier Márquez (2005). DIAGNÓSTICO Y ESPECIFICACIÓN DE MODELOS PANEL EN STATA 8.0, División de Estudios Políticos, CIDE, Mexico.

$*$ significant at $10 \%$; ** significant at $5 \%$; *** significant at $1 \%$

En base a los resultados de González, K., Yan, D., \& González, J. (2019), La ecuación de predicción para los modelos estudiados está dada de la siguiente forma:

$$
\begin{gathered}
P I B=-433.55+0.3162 P O-433.55 E I+188.285 \\
P I B=-457.32+0.3095 P O-0.00995 E I+226.09 \\
P I B=-491.470+126.494 P O-3.506 E I+85.470
\end{gathered}
$$

Donde la ecuación (17) es la ecuación de predicción para el modelo de regresión agrupada, la ecuación (18) es la ecuación de predicción para el modelo de efectos aleatorios y, por último, la ecuación (19), la ecuación para el modelo de efectos fijos. 
Dado el R cuadrado (R-squared) el modelo más acorde para el análisis de datos de panel, en este caso, es el segundo modelo, fixed effects o también conocido como método de efectos fijos, el coeficiente de determinación del mismo es mayor que la de los otros métodos, lo cual induce en un coeficiente de correlación (r) más alto y, por ende, un análisis más acertado al relacionar el PIB y la población ocupada (PO) y el empleo Informales (EI) para estudiar el impacto de los mismos en el país.

Para el modelo escogido, la ecuación de predicción corresponde a la ecuación (13), donde nuestra constante es -491.470, con un error estándar de 85.470. El R2 (Rsquared) es 0.9892 lo cual representa una determinación lineal moderada, que indica que el modelo de efectos aleatorios puede predecir el $98.92 \%$ del comportamiento del PIB en Panamá.

\section{Conclusiones}

$\mathrm{Al}$ analizar los resultados obtenidos en los tres modelos econométricos de datos de paneles, encontramos que el modelo más factible para llevar un estudio del Producto Interno Bruto (PIB) en Panamá es el modelo fixed effects cuyo (r) cuadrado es el más elevado, y facilita un estudio acertado al relacionar nuestras variables; por lo que, a futuras investigaciones de este sector, si se trabaja con este modelo, será más rápido conocer el efecto en la economía nacional.

Para el estudio de la investigación fue imprescindible conocer y entender el pensamiento Keynesiano, el cual se utiliza en las economías modernas; de igual manera cómo funciona y se maneja el mercado de bienes y el mercado laboral, teniendo en cuenta los datos proporcionados por la Contraloría General de la República de Panamá. Para futuros trabajos se recomienda utilizar herramientas como software para que la investigación no solo se base en teoría, sino que contenga mapas y gráficos, que puedan ser interpretados de manera clara y precisa tanto para el autor como para el lector. Con este artículo se busca conocer cuál es el aporte que brinda el Producto Interno Bruto (PIB) en Panamá, y la Población Ocupada (PO) y el Empleo Informal (EI), y qué medidas tomar de acuerdo al análisis obtenido, para generar mayores empleos que beneficien a la población en Panamá 


\section{Referencias bibliográficas}

Charles I. Jones (2000). Introducción al crecimiento económico /, 1a. ed. - México: Pearson Educación, 203 p.

González, J. (2017). La migración y el mercado trabajo local: caso español. años: 20012006. Guacamaya, 1(1), 41-84. Recuperado a partir de http://revistas.up.ac.pa/index.php/guacamaya/article/view/309

González, K., Yan, D., \& González, J. (2019). Estudio del producto interno bruto de hoteles y restaurantes y su efecto en el mercado laboral de Panamá. Guacamaya, 4(1), 20-37. Recuperado a partir de http://revistas.up.ac.pa/index.php/guacamaya/article/view/1038

Herrera, A., \& González, J. (2018). Estudio del transporte, almacenamiento y comunicaciones y su efecto sobre el crecimiento del producto interno bruto (pib) de la república de panamá. años: 2000-2015. Guacamaya, 2, 42-57. Recuperado a partir de http://revistas.up.ac.pa/index.php/guacamaya/article/view/110

Him, R., Ortega, L., \& González, J. (2019). Actividades inmobiliarias, empresariales y de alquiler, y su efecto en la economía de Panamá. Guacamaya, 3(2), 56-72. Recuperado a partir de http://revistas.up.ac.pa/index.php/guacamaya/article/view/438

Javier Aparicio y Javier Márquez (2005). DiAGNÓSTICO Y ESPECIFICACIÓN DE MODELOS Panel en Stata 8.0, División de Estudios Politicos, CIDE, México.

Pérez, J., Ashaw, M., Henríquez, T., \& González, J. (2019). Aplicaciones con OpenGeoda entre la Producción Interna Bruta Total (PIB) y la Población Económicamente Activa (PEA) en Panamá, Años 2007-2015. Guacamaya, 4(1), 38 - 64. Recuperado a partir de http://revistas.up.ac.pa/index.php/guacamaya/article/view/1039 